\title{
The effect of metabolism on the course of labor and the postpartum period in highly productive cows
}

\author{
Murat Baimishev 1,*, Sergey Eremin², Kirill Plemyashov ${ }^{3}$, Hamidulla Baimishev $^{1}$, Igor Konopeltsev $^{4}$, and Kairly \\ Yesengaliev $^{5}$ \\ ${ }^{1}$ Samara State Agrarian University, 446442 Kinel, Samara region, Russia \\ ${ }^{2}$ Nizhny Novgorod Agricultural Academy, 603107 Nizhny Novgorod, Russia \\ ${ }^{3}$ St. Petersburg Academy of Veterinary Medicine, 196084 St. Petersburg, Russia \\ ${ }^{4}$ Vyatka State Agricultural Academy, 610017 Kirov, Russia \\ ${ }^{5}$ West Kazakhstan Agroindustrial University named after Zhangir Khan, 090009 Uralsk, Republic of Kazakhstan
}

\begin{abstract}
The purpose of the research is to determine the etiopathogenesis of reproductive dysfunction in highly productive cows. For this, one group of cows was formed on the principle of paranalogs in the amount of 37 animals inseminated in the first sexual hunt after calving, followed by taking blood samples from them using the Monovet system, considering the duration of pregnancy. During the start-up period, blood was taken 1-4 days before calving and on the first day after calving. A total of 253 blood samples were examined. Subsequently, depending on the effectiveness of insemination, animals were divided into two groups. The first group included inseminated cows after the first insemination (20 animals), the second group included 17 unfertilized cows after the first insemination. Subsequently, blood was taken from animals considering the course of childbirth and the postpartum period. Blood counts were studied according to generally accepted methods using certified equipment. The study found that at an early stage of pregnancy, cows have a significant difference in lipid metabolism and in their peroxidation, in the state of antioxidant systems compared to unstable animals. In the process of pregnancy development in cows, there is a decrease in the level of total lipids and their class, and the accumulation of products of transoxidation of lipids is reduced. In animals with retention of the placenta, a low lipid metabolism and a higher level of peroxidation were established already in the dry period. After calving, this difference increases. The obtained data can be used to develop an algorithm for the prevention of postpartum complications in cows by using substances with antioxidant properties.
\end{abstract}

\section{Introduction}

The main task of intensifying dairy cattle breeding is to increase the level of animal milk production, which largely depends on the feeding conditions during preparation for calving and after calving. That is especially important for industrial milk production technology, since it requires preparation for insemination, calving not individual animals, and a large number of their analogues - by age, gestational age. When preparing animals for calving and in the newborn period, balanced feeding is an indispensable condition.

Feeding of pregnant animals has a great influence on the development of the fetus, especially in the second half of pregnancy, since intensive growth of the fetus occurs during this period. Therefore, when preparing highly productive cows for calving and subsequent lactation, it is important to establish a feeding level that ensures lipid metabolism, both for your own body and for creating a reserve of nutrients in the mother's body for the postpartum period and future lactation.
Microbial digestion of fiber and other nutrients in the gum stomachs of the ruminants indicates the nature of the influence of the formed and used metabolites of the substrate [1]. As a result of the relative values of some key processes, primarily lipogenesis in ruminants, it differs significantly from similar processes and nonruminant ones. The main precursor of high molecular weight fatty acids in ruminants is acetate $[2,3]$.

Therefore, cows, consuming a relatively small amount of lipids, about $5 \%$ of fat in energy metabolism, are much more dependent on non-glucose metabolites than monogastric animals. The importance of lipids is also manifested in the fact that they serve as the main form of energy storage in the body. The main metabolic function of adipose tissue in all placental animals is to remove lipids and lipid precursors from the blood with a caloric excess and release of fatty acids during periods of energy deficiency. This metabolic situation is very characteristic of cows, especially highly productive ones -2-3 months of lactation.

Even with all known nutritional balance requirements, cows lack energy and protein for milk

*Corresponding author: Baimishev_M@mail.ru 
production [3]. In this connection, there is a need to establish the boundaries of the maximum and optimal mobilization of reserve lipids in cows and find out the factors favorable to the use of fat reserves in the life of cows. Research in this area would allow a better understanding of the effect of tissue lipids on the general energy, the formation of milk and the function of cows' reproduction $[4,5]$.

Industrial technology for producing livestock products involves the constant adaptation of animals to the effects of various adverse stress factors caused by conditions of keeping, feeding, exploitation [6-9]. The most pronounced negative effect of such factors is exerted on pregnant cows, in the body of which increased energy consumption occurs, caused by intensive exploitation and development of the fetus $[10,11]$, which leads to the intensive use of plastic tissue compounds, primarily deposited fat, and hereinafter, structural lipids [4, 12, 13]

Mobilization of fatty acids occurs in complex oxidation reactions with carbohydrates and is often disrupted due to the relative lack of the latter. In this connection, under-oxidized compounds enter the bloodstream leading to depletion of the animal organism, vitamins, bioantioxidants arising due to high physiological load, stress, errors in feeding, which creates favorable conditions for the activation of nonenzymatic lipid oxidation $[3,5,7,12-15]$ which adversely affect the state of metabolism, and therefore the reproductive function of animals [16].

However, studies aimed at studying this issue in world and domestic literature are not enough. In the literature available to us there is no information about the effect of lipid metabolism and the antioxidant system of the body during pregnancy and the occurrence of obstetric pathology, which determined the topic of our research.

The goal of the research is to determine the etiopathogenesis of reproductive dysfunction in highly productive cows. To achieve this goal, the following tasks were performed:

- study of the lipid peroxidation and the state of the absorbent and thiol-disulfide redox system and the content of vitamins $\mathrm{A}$ and $\mathrm{E}$ depending on the physiological state;

- determination of the effect of the lipid spectrum of blood serum of dry cows on the course of labor and the postpartum period.

\section{Materials and methods}

The studies were conducted on cows of black-motley breed aged 3-5 lactations with a live weight of $480-520 \mathrm{~kg}$. For this, a group of cows was formed in the amount of 37 animals inseminated after calving in the first insemination, followed by regular blood sampling from them using the Monovet system in the following pregnancy periods: $4-7 ; 14-17 ; 24-34 ; 35-50 ; 88-95$; 195-210 days; during the launch period; $1-4$ days before calving and on the first day after calving. A total of 253 blood samples were examined. Subsequently, depending on the fruitfulness of insemination, the animals were divided into two groups. The first group included 20 cows fruitfully inseminated after the first insemination. The second group included 17 cows that did not fertilize after the first insemination. In the process of research, pregnant animals, for which clinical experience was confirmed during the periods of completion of fruiting, the course of childbirth, and the postpartum period, have confirmed the entire experiment. This group of animals was divided according to the results of the course of labor and the postpartum period into two groups. The first group includes animals with a normal physiological course of childbirth and the postpartum period ( 15 animals). The second group includes cows with a pathology of childbirth (retention of the placenta) and postpartum complications (postpartum endometritis) (5 animals).

The content of total serum lipids was studied using a unified method using the LAHEMA ready-made sets of Czechoslovakia, lipid extraction using the method of Folch followed by thin-layer chromatography on FIXION-50x8 plates and densitometry. Lipid peroxidation products (malondialdehyde, diene conjugates, triene conjugates) were studied by the method of Kitabchi and Bulge. The state of ascorbate and thiol disulfide redox systems was studied according to the method of V. Sokolovsky (1974). Determination of vitamin A and E - spectrophotometrically by the Bier method.

The obtained data of biochemical studies were interpreted by the method of retrospective analysis. All obtained digital material was processed biometrically using the computer program Microsoft Excel with determination of the difference criterion in percentage terms.

\section{Results}

As a result, the biochemical parameters of the blood of cows, depending on the productivity of insemination, had significant differences in several indicators (Table 1).

Thus, the content of total serum lipids in pregnant animals was $15.8 \%$ higher, compared with unfertilized animals. In the class composition of lipids, the most significant difference was found in the content of nonesterified fatty acids (NEFA), triglycerides and cholesterol, both free and ether bound. These differences are explained by the rather high energy costs in pregnant animals, necessary for the formation of the embryo, the development and growth of the fetus, and for maintaining the homeostasis of the mother's body. In non-pregnant animals, a decrease in free cholesterol (by $28.65 \%$ ), especially its ester-bound fraction (14.91\%), indicates a lower synthetic function of the liver, as evidenced by the low serum levels of vitamins $\mathrm{A}$ and $\mathrm{E}$ (a difference of 11.3 and $23.65 \%$ ).

In addition, a higher level of malondialdehyde (MDA) and diene conjugates of fatty acids - products of lipid peroxidation - characterizes an increased degree of the process of free radical oxidation. The content of hydroperoxide lipids or malondialdehyde in unborn cows 
was $2.85 \%$ higher than that of pregnant cows. The number of diene conjugates (relative optical units) per $1 \mathrm{ml}$ of plasma in unborn cows is more by $42.57 \%$, and the content of vitamin $\mathrm{E}$ (tocopherol) is less by $25.65 \%$.

The content of vitamin A in pregnant cows is 11.30 $\%$ higher compared to unrelated cows. The indices of the ascorbate system in oxidative forms are $7.89 \%$ higher in unborn cows, and the reducing forms in unborn cows are
$26.30 \%$ lower. The same tendency is observed in the thiol-disulfide antioxidant system.

A shift towards the oxidized forms of the components of both the ascorbate and thiol disulfide antioxidant systems, as well as the lower tocopherol content, indicate inadequate tension of the antiradical chains and a decrease in their buffer capacity, which negatively affects ovarian function [17-19].

Table 1. Biochemical blood parameters of pregnant and unfertilized cows

\begin{tabular}{|c|c|c|c|}
\hline \multirow{2}{*}{ Indicators } & \multicolumn{2}{|c|}{ Group of cows } & \multirow{2}{*}{ Difference in $\%$} \\
\hline & First (pregnant) & Second (non-pregnant) & \\
\hline Number of animals & 20 & 17 & \\
\hline Total lipids, mg \% & $426.10 \pm 23.40$ & $358.60 \pm 21.90$ & -15.84 \\
\hline Phospholipids, mg \% & $267.50 \pm 18.30$ & $259.00 \pm 18.70$ & -3.18 \\
\hline Free cholesterol, mg \% & $34.20 \pm 2.80$ & $24.40 \pm 3.20$ & -28.65 \\
\hline NEFA, mg \% & $44.70 \pm 3.00$ & $17.05 \pm 3.10$ & -61.91 \\
\hline Triglycerides, mg \% & $21.64 \pm 2.70$ & $3.74 \pm 1.90$ & -59.61 \\
\hline Esters of cholesterol, $\mathrm{mg} \%$ & $58.11 \pm 4.70$ & $49.44 \pm 4.20$ & -14.91 \\
\hline Lipid hydroperoxides (MDA), $\mu \mathrm{mol} / \mathrm{L}$ & $4.89 \pm 0.45$ & $5.03 \pm 0.62$ & +2.85 \\
\hline Diene conjugates (rel. Optical units per $1 \mathrm{ml}$ of plasma) & $1.48 \pm 0.21$ & $2.11 \pm 0.26$ & +42.57 \\
\hline Vitamin E, mg \% (tocopherol) & & $0.712 \pm 0.092$ & -25.65 \\
\hline Vitamin A, mcg \% & $42.30 \pm 2.90$ & $37.50 \pm 3.80$ & -11.30 \\
\hline \multicolumn{4}{|l|}{ Ascendant system: } \\
\hline (ox.form), mg \% & $0.38 \pm 0.06$ & $0.41 \pm 0.06$ & +7.89 \\
\hline (reduced form), mg \% & $0.39 \pm 0.05$ & $0.28 \pm 0.04$ & -26.30 \\
\hline \multicolumn{4}{|l|}{ Thiol Disulfide System: } \\
\hline (ox.form), mol/l & $22.00 \pm 1.80$ & $29.30 \pm 3.10$ & +33.18 \\
\hline (reduced forms), mol/1 & $32.80 \pm 2.30$ & $28.40 \pm 2.50$ & -13.42 \\
\hline
\end{tabular}

With an increase in the gestation period, some changes were detected in the lipid spectrum of the blood serum of pregnant animals. Thus, a decrease in the level of total lipids, unsterilized fatty acids and triglycerides was noted, which is quite typical for this physiological period of a gradual decrease in milk productivity and a still insignificant growth of the fetus. In the redox ascorbate and thiol disulfide systems, a slight increase in oxidized products with respect to reduced products and a slight increase in lipid peroxidation products were recorded.

In the dry period, the content of total lipids in animals without pathology was $3.42 \mathrm{~g} / \mathrm{l}$, which is $0.33 \mathrm{~g} / \mathrm{l}$ more than in animals with postpartum pathology. The serum phospholipid content in animals without pathology is $26.74 \mathrm{mg} \%$ higher than in animals with pathology. The same regularity in the content of lipids and phospholipids was noted before calving for 1-4 days and 1-2 days after calving. Before calving, it was found that an increase in non-sterilized fatty acids is without pathology.

During this period, an increase in triglycerides in cows with pathology was noted. The blood serum content of ether-cholesterol in the dry period in cows without pathology is $2.16 \mathrm{mg} \%$ higher. Before calving, this difference is $4.42 \mathrm{mg} \%$, and after calving, $3.52 \mathrm{mg}$ $\%$, which is apparently associated with the periods of calving, calving, which are a significant stress syndrome for the body of pregnant cows, which naturally affected the biochemical picture of the blood, which was expressed in a significant increase in products lipid peroxidation - malondialdehyde by $12-17 \%$, diene and triene conjugates - by $10-15$ and $8-15 \%$, respectively, and 1-4 days before calving in animals, which subsequently had a pathological course childbirth, in particular - detained the latter (5 animals) were characterized by an increased content in the blood plasma of lipid peroxidation products.

Thus, the difference in the number of dienes, trienes, and malondialdehyde products was, compared with animals without obstetric pathology, 14.00, 12.00, and $23.50 \%$, respectively. In the first days after calving, this difference increased even more. Cows with retention of the afterbirth had a significantly higher level of lipid peroxidation products and were characterized by a predominance of oxidized products over the antiradical thiol disulfide and ascorbate systems restored in the antioxidant balance. The content of vitamins $\mathrm{A}$ and $\mathrm{E}$ in these animals both during the starting period, several days before calving, and after calving was significantly lower (on average by $12-18 \%$ ).

Changes in the lipid spectrum of blood serum of pregnant cows during the start-up period, shortly before calving and immediately after calving, are presented in table 2 .

As follows from the data presented, animals with retention of the placenta had a lower serum lipid content and, accordingly, all their classes. Moreover, already during the start-up period, the amount of total lipids in cows with obstetric pathology amounted to $90.30 \%$ of the level of normally calving cows, before calving $85.40 \%$, and after calving, respectively $87.00 \%$. A similar pattern is noted for the class of phospholipids.

In cows with afterbirth retention, the number of phospholipids after calving had an even greater difference $(85.50 \%$, compared with animals without obstetric pathologies). The fraction of cholesterol, both 
free and ether-bound, in normally calving cows in all the above periods was superior to that of cows with delayed afterbirth.

The most pronounced difference was recorded 1-4 days before calving in the class of unbound cholesterol. The level of this metabolite in the indicated period in animals with obstetric pathology amounted to $79 \%$ of healthy animals. In animals with a normal course of calving, childbirth was characterized by a more intensive preparatory period, as indicated by a significant mobilization of energy reserves from the fat depot 1-4 days before calving in animals without deviations in the birth period. This was expressed in a marked decrease in triglycerides and an increase in unesterified fatty acids. In animals with retention of the placenta, energy training was less intense.

Table 2. Biochemical indicators of lipid metabolism depending on the manifestation of birth and postpartum pathology

\begin{tabular}{|c|c|c|c|c|}
\hline \multirow{2}{*}{ Indicators } & \multirow{2}{*}{$\begin{array}{c}\text { Childbirth } \\
\text { and the postpartum period }\end{array}$} & \multicolumn{3}{|c|}{ Physiological periods } \\
\hline & & dry & before calving in $1-4$ days & $1-2$ days after calving \\
\hline \multirow{3}{*}{ Total lipids, g/l } & without pathology & $3.42 \pm 0.22$ & $3.38 \pm 0.25$ & $3.02 \pm 0.23$ \\
\hline & with pathology & $3.09 \pm 0.27$ & $2.99 \pm 0.11$ & $2.63 \pm 0.08$ \\
\hline & difference in \% & -9.65 & -16.54 & -12.92 \\
\hline \multirow{3}{*}{$\begin{array}{l}\text { Phospholipids, } \\
\text { mg \% }\end{array}$} & without pathology & $233.97 \pm 20.19$ & $227.87 \pm 17.87$ & $208.49 \pm 18.96$ \\
\hline & with pathology & $207.23 \pm 13.47$ & $201.05 \pm 17.18$ & $178.45 \pm 5.47$ \\
\hline & difference in $\%$ & -11.43 & -11.77 & -14.41 \\
\hline \multirow{3}{*}{$\begin{array}{l}\text { Free cholesterol, } \\
\text { mg } \%\end{array}$} & without pathology & $22.29 \pm 1.62$ & $22.30 \pm 3.61$ & $18.58 \pm 1.71$ \\
\hline & with pathology & $19.21 \pm 2.34$ & $17.65 \pm 1.57$ & $15.81 \pm 1.25$ \\
\hline & difference in $\%$ & -13.82 & -28.60 & -14.91 \\
\hline \multirow{3}{*}{ NEFA, mg \% } & without pathology & $19.36 \pm 2.36$ & $24.75 \pm 2.02$ & $19.02 \pm 2.65$ \\
\hline & with pathology & $20.71 \pm 1.85$ & $22.64 \pm 1.88$ & $20.58 \pm 1.49$ \\
\hline & difference in $\%$ & +6.97 & $-8,86$ & +8.20 \\
\hline \multirow{3}{*}{$\begin{array}{l}\text { Triglycerides, } \\
\mathrm{mg} \%\end{array}$} & without pathology & $10.42 \pm 1.23$ & $8.96 \pm 1.13$ & $9.37 \pm 1.05$ \\
\hline & with pathology & $9.16 \pm 0.99$ & $10.76 \pm 2.01$ & $8.32 \pm 1.03$ \\
\hline & difference in \% & -12.10 & +20.08 & -11.21 \\
\hline \multirow{3}{*}{$\begin{array}{l}\text { Esters of } \\
\text { cholesterol, mg } \\
\%\end{array}$} & without pathology & $48.93 \pm 2.23$ & $51.79 \pm 6.57$ & $43.75 \pm 3.69$ \\
\hline & with pathology & $46.67 \pm 5.01$ & $47.37 \pm 4.14$ & $40.23 \pm 2.39$ \\
\hline & difference in \% & -4.62 & -8.54 & -8.05 \\
\hline
\end{tabular}

Note: without pathology - blood counts of cows with normal calving: 15 anim; with pathology - with trail retention and endometritis (5 anim).

\section{Conclusion}

According to domestic and foreign researchers, pregnancy and the birth that follows, restoration of the reproduction function after childbirth in mammals is accompanied by changes in metabolism to hidden forms of its violation. The tension of metabolic processes manifests itself towards the end of pregnancy and in the early postpartum period. From the above experimental data, the occurrence of obstetric pathology, impaired reproduction function is largely determined by the process of lipid peroxidation. The processes of lipid peroxidation play an important role in the normal physiology and biochemistry of the cell, but also act as a nonspecific link in the development mechanisms of various pathologies, and depending on the level of their activity, they can turn into the leading link in the pathogenesis of the disease.

In the process of pregnancy development in cows, there is a decrease in the level of total lipids, their classes, and there is also a tendency to accumulation of lipid peroxidation products. Cows at the stage of early pregnancy have significant differences in lipid metabolism, their peroxidation and the state of antioxidant systems, compared with unfertilized animals, which is expressed in a sufficiently high content of lipids of various classes, a low level of their peroxidation and a rather significant capacity antioxidant systems.
With an increase in the duration of pregnancy, changes were detected in the lipid spectrum of the blood serum of pregnant cows, a decrease in the level of total lipids, unesterified fatty acids and triglycerides was noted, which is associated with a decrease in the level of milk productivity and growth of the fetus.

During this period, in the redox ascorbate and thiol disulfide systems, a slight increase in the oxidized products was recorded relative to the reduced ones, and a slight increase in the products of lipid peroxidation was observed. During the start-up of animals, a significant increase in the products of lipid peroxidation, malondialdehyde to $17.00 \%$, diene and triene conjugates, respectively, to $15.00 \%$, was found. 4 days before calving, in animals that subsequently had a delay in the afterbirth, the content of lipid peroxidation products in the blood plasma increased. The difference between animals with pathology and without pathology of childbirth ranged from 12.00 to $23.00 \%$.

In cows with retention of the placenta already in the dry period they are characterized by a lower energy level of metabolism, lipid metabolism, and a higher level of lipid peroxidation. According to the class of phospholipids in cows with retention of the afterbirth, the number of phospholipids is significantly less in comparison with animals without obstetric pathology. The fraction of cholesterol, both free and ether-bound in normally calving cows in all periods exceeded that with retention of the afterbirth. 
In this connection, it is necessary to consider that these processes proceed in conjunction with the antioxidant system of the body and its insufficiency is accompanied by activation of lipid peroxidation. The data obtained indicate that the nature of lipid peroxidation and the state of the antioxidant defense system of the body can be used for timely correction of lipid metabolism and antioxidant system disorders in cows, but also serve as a criterion indicator of impaired reproductive function of highly productive cows, which will predict diseases and obstetric pathology.

\section{References}

1. P.G. Toral, G. Hervas, V. Peiro, P. Flutos, Animals, 8(11), 196 (2018)

2. Y. Zhu, G. Liu, X. Du, Z. Wang, X. Li. J. of Dairy Sci., 102(2), 1725-1735 (2019)

3. V.D. Convay, M.V. Zabolotnykh, Bull. of the Omsk State Agrar. Univer., 3(27), 130-136 (2017)

4. P.A. Krasochko, N.M. Avlasko, Veterinary medicine on the path of innovative development (Grodno, 2016)

5. M.H. Baimishev, S.P. Eremin, H.B. Baimischev, V.V. Zemlyankin, H.A. Safiullin, J. of Pharmaceut. Sci. and Res., 10(4), 819-823 (2018)

6. N.V. Belugin, N.A. Pisarenko, A.V. Konobeysky, B.V. Pianov, E.N. Shuvalova, Bull. of the AgroIndustr.1 Complex of Stavropol Territ., 2(14), 112-116 (2014)
7. I.I. Kalyuzhny, N.D. Barinov, A.V. Korobov, Metabolic disorders in highly productive cows (Saratov, 2010)

8. A.V. Trebuhov (Barnaul, 2012)

9. X. Du, T. Shen, H. Wang, G. Liu, X. Li. J. of Dairy Sci., 101(10), 9544-9558 (2018)

10. E.V. Dushkin, E.N. Belogubova, Actual problems and methodological approaches to the diagnosis, treatment and prevention of animal diseases (Donskoy GAU, 2016)

11. S. Yu, Smolentsev. Veterinarian, 2, 40-44 (2016)

12. Y. Li, H. Ding, J. Dong, X. Li, J. of Cellular Physiol., 234(5), 6054-6066 (2019)

13. S. Maqsood, A. Al-Dowalia, P. Mudgil, B. Jobe, H.M. Hassan, Food Chemistry, 279, 328-338 (2019)

14. A.M. Gertman, E.M. Rulikova, T.S. Samsonova, Scientific support for the innovative development of agribusiness (Ufa, 2010)

15. A.V. Trebuhov, A.A. Elenschleger, Veter., 5, 58-62 (2016)

16. H. Baimischev, M. Baimischev, V. Grigoryev, I. Uskova, I. Hakimov, Asian Pacific J. of Reproduct., 7(4), 167-171 (2018)

17. J. Zhang, G. Wang, C. Zhao, Z. Fan, C. Xia, The Veter. quarterly, 38(1), 47-52 (2018)

18. A.M. Gertman, D.M. Maksimovich, T.S. Samsonova, Actual problems of electrophysiology and non-infectious animal pathology (Ulan-Ude, 2009)

19. R. Mesilati-Stahy, N. Agrov-Agroman, PLoS ONE, 13(12), 209-565 (2018) 